\title{
ESTADOS UNIDOS: CRISIS ECONÓMICA, REELECCIÓN PRESIDENCIAL Y POLARIZACIÓN POLÍTICA*
}

The United States: Crisis, Reelection, and Polarization

\author{
JULIETA SUÁREZ-CAO \\ Pontificia Universidad Católica de Chile
}

\begin{abstract}
RESUMEN
El presente artículo desarrolla los temas principales del año 2012 en los Estados Unidos. Se describen la crisis económica y los principales asuntos de política doméstica e internacional. La mayor atención, sin embargo, se concentra en el acontecimiento más importante del año: las elecciones generales. Se relevan así tanto aspectos de la campaña electoral como de los resultados de los comicios, que culminan con la reelección del presidente Barack Obama. Asimismo, se propone una interpretación del triunfo demócrata y del gobierno dividido resultante. Finalmente, se sostiene que los fenómenos de la polarización política, la transformación del elector medio estadounidense, la coalición de apoyo de Obama y el efecto del redistritado en la cámara baja son puntos clave para entender los resultados de la elección.
\end{abstract}

Palabras clave: Elecciones 2012, Encuestas, Crisis económica, Colegio Electoral, Polarización.

\begin{abstract}
The present article provides a summary of the most important political events in the United States during 2012. It offers a discussion of the economic crisis and the main domestic and international issues. The focus of attention, however, is on the most relevant event of the year: the general elections that led to the reelection of Barack Obama as well as on the electoral campaign and election results. I advance an interpretation of the Democratic victory and the resulting divided government based on key notions about political polarization, the transformation of the American median voter, the social coalition behind Obama's reelection, and the effect of gerrymandering on congressional districts.
\end{abstract}

Key words: 2012 General Elections, Surveys, Economic Crisis. Electoral College, Polarization.

* La autora agradece los comentarios de un referí anónimo así como al Núcleo Milenio para la Estatalidad y la Democracia en América Latina (NS 100014) por el apoyo prestado para la elaboración de este artículo. 


\section{INTRODUCCIÓN}

El año 2012 estuvo marcado por acontecimientos importantes en diversas materias para los Estados Unidos de América. Además de la reelección del primer presidente afroamericano, Barack Obama, comenzó una lenta recuperación de la crisis económica más importante de lo que va del siglo. Con un descenso del desempleo y un crecimiento moderado, las políticas de estímulo empezaron a dar un poco de respiro a la acuciante situación económica del país norteamericano.

El presente anuario se centra en el acontecimiento más relevante de 2012: las elecciones presidenciales y legislativas. Estos comicios revirtieron la tendencia ganadora del Partido Republicano en las elecciones intermedias de 2010. En esa ocasión, los demócratas perdieron la mayoría en la Cámara Baja en una elección que fue leída como no solo el triunfo del partido opositor, sino como el triunfo de una facción al interior del mismo que presenta visiones muy conservadoras en materia económica, social y cultural: el movimiento del Tea Party. Por el contrario, en 2012, el electorado estadounidense refrendó la administración demócrata, pero mantuvo un congreso dividido con los republicanos controlando la Cámara de Representantes y los demócratas el Senado federal.

La polarización de las élites políticas jugó un rol muy importante en el desarrollo de la campaña y las elecciones, en especial dentro del Partido Republicano. Si bien el Tea Party logró capitalizar el descontento de buena parte de los votantes en las elecciones anteriores, en 2012 sus posturas extremas en asuntos de alta visibilidad (por ejemplo, la reforma migratoria) le restaron apoyo de sectores que demostraron ser claves como el electorado latino. Las elecciones de 2012 presentaron entonces un límite claro al rédito político de posturas políticas más extremas. En el país donde en el siglo pasado se hacía un llamado a tener partidos políticos más "responsables" que presentaran alternativas claras de gobierno, el abandono progresivo de posturas moderadas por parte de los republicanos fue penalizado en las urnas. ${ }^{1}$

Otro aspecto interesante de las elecciones de 2012 estuvo marcado por lo que se conoce como horse race journalism (Nisbet 2008), esto es, un tipo de cobertura de las elecciones por parte de los medios de comunicación que emula el relato de una carrera de caballos. Así, se tiende a enfatizar la incertidumbre acerca del resultado final a través de la presentación de encuestas y del análisis de los formadores de opinión (pundits). ${ }^{2}$ Los medios de comunicación, en este caso, se focalizaron en sondeos nacionales que pronosticaban que ambos candidatos estaban dentro de margen de error y que por ende el resultado de la elección era incierto. Por otro lado, los expertos en estadísticas aplicadas a encuestas que venían siguiendo la evolución de las encuestas estatales, que son las relevantes en

1 En 1950, la Comisión de Partidos Políticos de la Asociación de Ciencia Política Americana publicó un reporte con un llamado a la necesidad de contar con partidos más modernos, programáticos y con ideologías diferenciadas -véase el documento original (1950) y los artículos de Ranney (1951) y Schlesinger (1951), así como sus derivaciones posteriores (Kirkpatrick 1971; Pomper 1971; Lowi 1983). Para un análisis a partir de este marco de los cambios de las últimas décadas consúltese Paulson (2007).

2 Un pundit es un analista experto en política, pero últimamente se ha asociado este término a una connotación negativa más cerca de lo que entenderíamos como un "opinólogo". 
sistemas de votación como el Colegio Electoral, daban a Obama como ganador con unas probabilidades altas de ganar la contienda. ${ }^{3}$ Estas elecciones serán recordadas como el triunfo de los expertos por sobre los opinólogos.

El presente artículo realizará un anuario de lo acontecido en los Estados Unidos en 2012 centrándose en los comicios generales. Así, la próxima sección expone los temas salientes de la realidad nacional y su impacto político, distinguiendo además aspectos de la coyuntura internacional. En la tercera sección se pone un énfasis especial en la campaña y en los problemas que concitaron una atención mayor por parte de los medios de comunicación y los políticos. La cuarta sección incluye los resultados de las elecciones generales que culminaron con la victoria del candidato del Partido Demócrata y análisis de las contiendas presidenciales, legislativas y subnacionales. La quinta sección resume los aspectos más relevantes del artículo y especula sobre el futuro político del país.

\section{COYUNTURA SOCIOECONÓMICA E INTERNACIONAL}

La crisis económica que afectó a los Estados Unidos desde 2007 es considerada como la peor desde la Gran Depresión de 1929. La crisis actual fue desatada por la burbuja en el mercado inmobiliario y la insolvencia de los créditos hipotecarios riesgosos. Un año más tarde, con la quiebra de una empresa financiera importante, Lehman Brothers, el pánico en los mercados financieros globales llevó a la intervención del gobierno de Estados Unidos (Helleiner 2011). El impacto político de la crisis fue amplificado al ocurrir en un año de elecciones generales como 2008. El gobierno del presidente saliente, George W. Bush, ordenó la absorción de las empresas crediticias conocidas como Fannie Mae y Freddie Mac y llevó a una suspensión de la campaña electoral del candidato republicano John McCain por unos días para trabajar en el Congreso, en su rol de senador por Arizona, en un plan de rescate financiero.

A partir de 2008, la administración Obama implementó programas de estímulo fiscal para reactivar la economía. Como se ilustra en el Gráfico 1, en 2010 se empieza a ver una lenta recuperación que se mantiene relativamente estable hasta la fecha. En 2012, la economía estadounidense creció un 2,2 por ciento con respecto al año anterior. Del mismo modo, se consiguió un leve descenso de los niveles de desempleo, que están actualmente por debajo del ocho por ciento luego de haber alcanzado un máximo de 9,6 en 2010.

Si bien la situación ha mejorado respecto de años anteriores, este desempeño no ha sido suficiente para declarar que la economía se encuentra ya recuperada. ${ }^{4}$ Como se discutirá en la próxima sección, la lenta mejora económica no impidió que la crisis fuera uno de los temas más sobresalientes de la campaña presidencial de 2012. En efecto, la necesidad de seguir aumentando el empleo y la disputa entre las dos visiones encontradas acerca de cómo lograr la recuperación económica propuestas por demócratas y republicanos

3 El más famoso de ellos es el Nate Silver, desde su blog Five Thirty Eight en el periódico New York Times http:/ / fivethirtyeight.blogs.nytimes.com/.

4 Para un análisis de la situación actual y los desafíos a futuro de la economía de los Estados Unidos, véase el estudio de la Organización de Cooperación y Desarrollo Económico (OECD 2012). 
Gráfico 1: Producto Bruto Interno: Cambio porcentual en relación al período precedente, por trimestres (2009-2012)

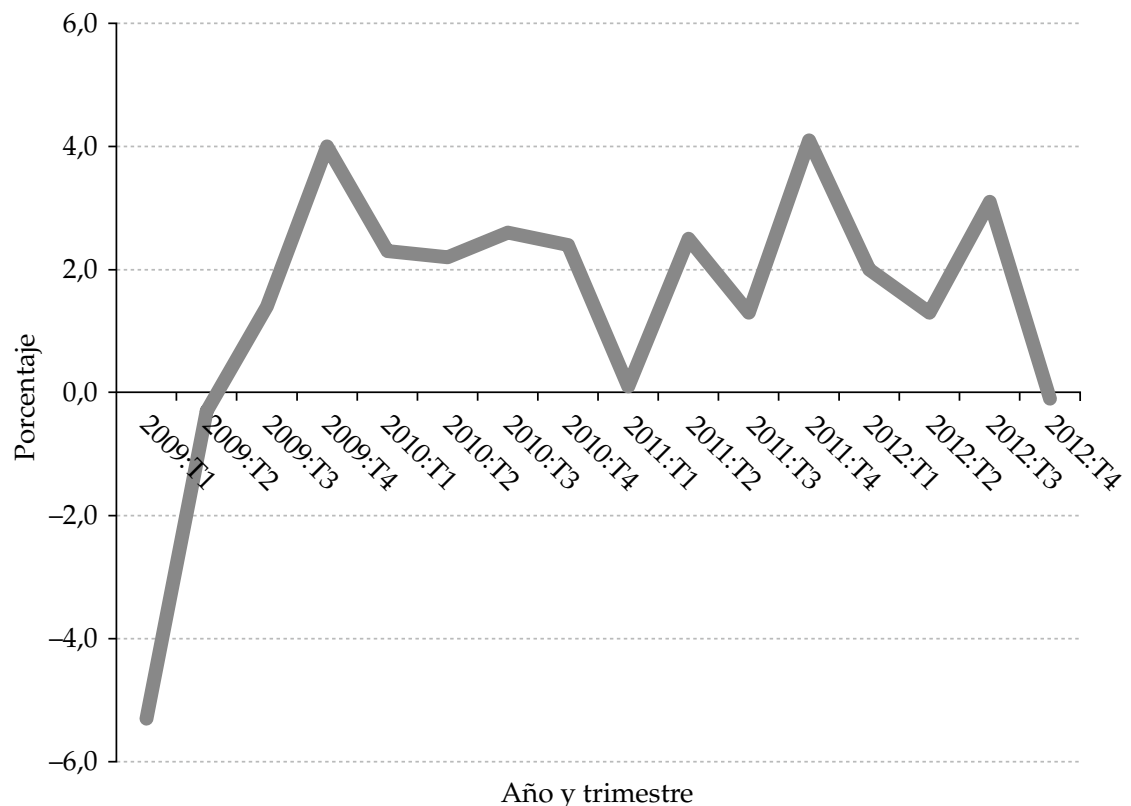

Fuente: Elaboración propia a partir de datos de Bureau of Economic Analysis, disponibles en http:/ / www.bea.gov/newsreleases/national/gdp/2013/pdf/gdp4q12_adv.pdf [Consulta: 23-1-2013].

dominaron los debates preelectorales. Esto no es solo porque la economía es un aspecto crucial de la vida social moderna, sino porque la situación económica suele ser el mejor predictor de la suerte electoral de los candidatos, en especial en un año como 2012 donde el actual presidente se presenta a su reelección. Se presume que el votante utiliza las contiendas electorales para premiar o penalizar el manejo económico de la administración vigente mediante la evaluación retrospectiva de su desempeño. Como ha venido sosteniendo la literatura acerca del voto económico en ciencia política: "el ciudadano vota por el gobierno si la economía está bien, si no lo está elige a la oposición" (Lewis-Beck y Stegmaier, 2000: 183).

Respecto de cuestiones de política doméstica, además del estado de la economía, uno de los acontecimientos más relevantes del año fue la decisión de la Suprema Corte de Justicia Federal de ratificar la ley del seguro de salud obligatorio impulsado por Obama en su primer mandato con el Acta de Cuidado de Salud de Bajo Precio (The Affordable Care Act (ACA)). En la opinión de The New York Times, esta ratificación fue "la más importante desde el New Deal en lo concerniente a asuntos relativos a las jurisdicciones del federalismo" (Liptak 2012). El punto a definir era si el gobierno federal estaba constitucionalmente autorizado para exigir que los habitantes posean un seguro de salud. La decisión judicial se definió por un margen estrecho de cinco contra cuatro votos y 
dictaminó que el mandato era constitucional. Las bases de la opinión judicial se asentaron sobre el derecho que tiene el Congreso de crear impuestos, ya que se interpretó que la multa a quienes no tuvieran un seguro de salud consistía en una especie de gravamen.

Otro de los acontecimientos más importantes de política doméstica se dio hacia finales de año cuando se produjeron negociaciones frenéticas para detener el "abismo fiscal" (fiscal cliff) que se produciría en 2013 cuando vencieran automáticamente ciertas provisiones que ahondarían el déficit fiscal, como la baja de impuestos sancionada durante la administración de George W. Bush. Si esto ocurriera, el pronóstico señalaba que se incrementaría tanto el déficit como el desempleo y se volvería a entrar en una recesión económica. ${ }^{5}$ La legislación impulsada por la Casa Blanca preveía mantener los recortes impositivos favorecidos por la anterior administración republicana con la excepción de aquellos que beneficiaban a los contribuyentes de más altos ingresos. El año nuevo comenzaría con el Congreso aprobando la American Taxpayer Relief Act (El Acta de Alivio a los Contribuyentes Estadounidenses) gracias a los esfuerzos conjuntos del Vicepresidente Joseph Biden y del líder republicano del Senado, Mitch McConnell (Kumar y Douglas, 2012). Esta ley evitaría el abismo fiscal al prorrogar los recortes impositivos que expiraban en 2013.

El pueblo de los Estados Unidos también tuvo que hacerle frente en 2012 a un desastre natural importante. El huracán Sandy azotó la costa este del país dejando un número total de ochenta y siete fallecidos. ${ }^{6}$ Asimismo, vastas regiones del país permanecieron por días sin suministro eléctrico ni de agua potable. Que la tormenta haya ocurrido quince días antes de las elecciones generales provocó mucha controversia sobre el potencial uso político de la tragedia. Se especuló, por ejemplo, acerca de si el impacto político incluiría una disminución de los votantes anticipados (early voting). ${ }^{7}$ Del mismo modo, se debatía si el presidente Obama sería capaz de capitalizar el desastre gracias a su postura ampliamente conocida en el debate por el calentamiento global: durante su primera presidencia, se impusieron estándares para regular las emisiones industriales de contaminantes y estimuló una transición a fuentes de energía más sustentables. ${ }^{8}$ Esta discusión cobraba sentido en el contexto de que el candidato del Partido Republicano, Mitt Romney, había propuesto en 2011 eliminar la agencia federal de gestión de emergencias (FEMA -Federal Emergency Management Agency) para achicar gastos del estado (Rosenthal, 2012).

Otras tragedias, pero producidas por seres humanos, fueron las matanzas en Colorado y Connecticut. ${ }^{9}$ En el estado del sudoeste, un tirador asesinó a doce personas en el estreno

$5 \quad$ Véase el documento de la Oficina de Presupuesto del Congreso (2012).

6 Las cifras están disponibles en NOOA (National Oceanic and Atmospheric Administration), la agencia norteamericana de la administración oceánica y atmosférica del servicio nacional del clima (Blake et al. 2013).

7 En algunos estados, los electores pueden votar antes del día de la elección, ya sea por correo o en persona.

8 Información del website de Barack Obama, disponible en http://www.barackobama.com/environment [Consulta: 28-2-2013].

9 El Washington Post reporta doce asesinatos de masas realizados por un tirador en 2012 (“U.S. mass shootings in 2012", disponible en http:/ / www.washingtonpost.com/wp-srv/special/nation/us-mass-shootings-2012). Los mencionados arriba fueron los incidentes en los que más personas resultaron muertas. 
de una película de la saga de Batman; mientras que en la costa este, otro hombre mató a veinte niños y seis adultos en un jardín infantil. Estos actos de violencia pusieron sobre el tapete la discusión del control de armas. Este es un viejo debate en los Estados Unidos que se suscita periódicamente sobre la constitucionalidad de la restricción en la compra y posesión de armas de guerra. En este caso, las repercusiones continúan ocupando espacios importantes en los medios de comunicación y en la agenda de la administración Obama que aboga por leyes más estrictas (Applebome y Santora, 2013; Healy, 2013), mientras que los republicanos suelen desaprobar cualquier tipo de regulación de lo que perciben como un derecho constitucional explicitado en la Segunda Enmienda a la Constitución de los Estados Unidos. ${ }^{10}$

Respecto de la coyuntura internacional, la primera administración de Barack Obama también tuvo que lidiar con las guerras en Irak y Afganistán. El retiro de tropas de Irak y el asesinato de Osama Bin Laden en Pakistán focalizaron la acción bélica estadounidense en Afganistán con miras a derrotar a la organización terrorista responsable por los ataques del 11 de septiembre de 2001, Al Qaeda. En 2012, sin embargo, la atención de la política exterior se concentró en otro lugar del globo: Medio Oriente. La guerra civil en Siria, el asesinato del embajador estadounidense y otros diplomáticos en Libia, las elecciones en Egipto, la tensión creciente con Irán y el deterioro de las relaciones con Israel fueron los acontecimientos internacionales del año (Cannon, 2012).

Las elecciones en Egipto donde se impuso la Hermandad Musulmana y la guerra civil en Siria parecen clausurar los últimos estertores de los movimientos de resistencia civil a las dictaduras en el mundo árabe que habían comenzado a fines de 2010. El rol de los Estados Unidos bajo la administración Obama en estas revoluciones ciudadanas fue de un apoyo por lo menos tácito y se evidenció en las palabras del presidente acerca de alentar el establecimiento de los derechos de libertad de expresión, de reunión, de credo, de libre elección de autoridades y de igualdad entre hombres y mujeres; así como favorecer las reformas económicas y políticas en la región. ${ }^{11}$ Similares posturas en beneficio del "poder blando", la diplomacia y la negociación fueron elaboradas por Obama acerca de la relación de Estados Unidos con Irán durante los debates presidenciales.

\section{ELECCIONES GENERALES 2012: LA CAMPAÑA ELECTORAL}

Sin dudas el acontecimiento del año fueron las elecciones presidenciales y legislativas. El Partido Demócrata llevó como candidato al presidente en ejercicio, mientras que el Partido Republicano seleccionó al suyo mediante un proceso de primarias y una convención partidaria. Los precandidatos republicanos que mantuvieron sus campañas después de las primeras elecciones fueron el ex-gobernador de Massachusetts Mitt Romney, el congresista libertario Ron Paul, el ex-presidente de la Cámara de Representantes Newt Gringrich y el ex-senador por Pennsylvania Rick Santorum. La convención del partido

10 El texto de la Segunda Enmienda sostiene que “...el derecho de los ciudadanos a poseer y llevar armas no será violado".

11 Discurso de Obama en el Departamento de Estado el 19 de mayo de 2011, citado en Keiswetter (2012). 
terminó eligiendo a Mitt Romney como candidato quien se había impuesto en la amplia mayoría de los estados y ganado el voto popular. ${ }^{12}$ Romney escogió como compañero de fórmula a un congresista por el estado de Wisconsin que contaba con el aval del Tea Party Movement, Paul Ryan (Shear, 2012). ${ }^{13}$

El Tea Party Movement es un movimiento ciudadano conservador nacido en 2008 que ha logrado, por un lado, revitalizar al conservadurismo y, por el otro, polarizar al Partido Republicano y a la sociedad estadounidense. Ha tomado el nombre de un hecho histórico fundacional en los Estados Unidos que fue el Boston Tea Party, cuando un grupo de patriotas tiraron al mar el té que venía de Inglaterra en un acto de protesta contra la política arancelaria del imperio inglés en 1773. Este acontecimiento sería uno de los disparadores de la revolución e independencia estadounidense. Así, el Tea Party Movement tiene en sus orígenes en la aversión contra los impuestos percibidos como una intromisión del gobierno federal en las libertades individuales. Como muchos movimientos, sin embargo, carece de una ideología precisa y presenta rasgos conservadores (por ej., en contra de la injerencia del gobierno en la economía), libertarios (por ejemplo, en contra de la intervención estadounidense en el extranjero) y populistas (por ej., posturas antiélite y antiintelectual). ${ }^{14}$

El éxito republicano en las elecciones intermedias de 2010, en las cuales consiguió la mayoría en la Cámara de Representantes, se debe en parte a la revitalización conservadora que trajo consigo el Tea Party Movement. Vanessa Williamson, Theda Skocpol y John Cuggin sostienen que hay poco de nuevo en el movimiento. En efecto, en su opinión, el mismo es la "nueva encarnación de tendencias de larga data en el conservadurismo americano" (2011: 26). Lo que estos autores encuentran de novedoso en el movimiento es su organización estructurada en redes de base (grass-roots networks). A partir de un estudio realizado con metodologías múltiples -desde el análisis de datos de encuestas hasta entrevistas etnográficas-, Williamson, Skocpol y Cuggin logran caracterizar a los tea partiers como una minoría compuesta principalmente por hombres blancos de mediana edad y de clase media alta: "entre el 55 y el 60 por ciento de los miembros son hombres; el 80-90 por ciento son blancos y el 70-75 por ciento son mayores de 45 años" (2011: 27). Estos datos no son menores, puesto que dichas características demográficas coinciden al dedillo con el perfil de los ciudadanos estadounidenses que tienden a participar en las elecciones (Wolfinger y Rosenstone, 1980).

La campaña electoral entonces se da en un contexto en el cual los republicanos ganaron las elecciones intermedias y vienen con un ímpetu ideológico revitalizado gracias al Tea

12 En estas primarias, Romney ocupó el primer lugar en treinta y siete estados y obtuvo el 52,61\% de los votos (Atlas Electoral de Dave Leip, Dave Leip's Atlas of U.S. Presidential Elections. Disponible en http:/ / uselectionatlas. org/RESULTS/national.php? $\mathrm{f}=0$ \&year=2012\&elect=2) [Consulta: 10-2-2013].

13 Esta elección fue similar a la que hizo John McCain cuatro años antes al nominar como candidata a la vicepresidencia a Sarah Palin en que ambos, Palin y Ryan, apelan al ala más conservadora del Partido Republicano, mientras que Romney y McCain son percibidos como más moderados. Adiferencia de Palin, sin embargo, Ryan es considerado como un experto en política pública (Strassel 2012; Zeleny y Rutenberg 2012).

14 Para una discusión exhaustiva del movimiento, véase la serie especial sobre el Tea Party de la Radio Pública Nacional (National Public Radio -NPR) disponible en http:/ /www.npr.org/series/130538063/the-tea-partyin-america [Consulta: 8-2-2013]. 
Party. Pero una elección presidencial no necesariamente se puede predecir a partir de una elección legislativa anterior; estas son contiendas diferentes en las que distintas cosas son las que están en juego. Sin lugar a dudas, en esta campaña el lugar predominante estuvo ocupado por la crisis económica anteriormente reseñada. A pesar de la recuperación lograda por la administración Obama, esta no había sido lo suficientemente dinámica para dejar el tema fuera de la agenda. Aunque tanto los demócratas como los republicanos coincidían en que la crisis aún no había terminado, las recetas que proponían para salir de la misma eran diametralmente opuestas. Los demócratas confían en las políticas de estímulo para reactivar la economía y el empleo, así como en que los más ricos paguen más impuestos. Los republicanos, por el contrario, propugnan que los más ricos tengan ventajas impositivas porque son el sector dinámico de la economía, para incentivarlos a que inviertan más y que se genere más empleo.

Otros temas de política doméstica que fueron clave en la campaña son la cuestión de la inmigración, el seguro de salud universal y los derechos para las minorías sexuales y de salud reproductiva. En estos ámbitos tampoco coincidían los demócratas y los republicanos. Los primeros dicen estar a favor de la amnistía a los inmigrantes ilegales y en contra de las deportaciones masivas (a pesar de esto, la primera administración de Obama avanzó poco en el terreno de la reforma migratoria), proponen sostener el sistema de salud universal recientemente ratificado por la Corte, están en contra de definir el matrimonio como la unión entre un hombre y una mujer (pero creen que esto debe ser un asunto de injerencia estatal y no federal) y asegurar el acceso a anticonceptivos y cubrir los costos de los abortos en determinados casos. Por su parte, los republicanos se muestran en contra de la amnistía y a favor de las deportaciones, buscan derogar el llamado Obamacare (ACA), proponen prohibir el matrimonio entre personas del mismo sexo a nivel nacional y consideran que el fallo que permitió el aborto a nivel federal (Roe v. Wade 1973) debería ser derogado y dejar a los estados definir la política al respecto. ${ }^{15}$

En relación con la política internacional, Obama continúa favoreciendo las negociaciones y la diplomacia por sobre la acción unilateral, ofreciendo apoyo a los movimientos de liberación en Medio Oriente dentro de una coalición con otras naciones y manteniendo una postura menos afín a los intereses de Israel que la que solía sostener Estados Unidos en el pasado. Los republicanos claman por retomar el liderazgo internacional que caracterizaba al país. Romney sostuvo en un discurso ante veteranos de guerra que él pretendía encabezar un siglo americano en el cual "Estados Unidos liderara el mundo libre, y el mundo libre liderara el resto del mundo", ya que él cree que "[Estados Unidos] es la fuerza del bien más grande que el mundo haya conocido y que nuestra influencia es tan necesaria como siempre". ${ }^{16}$ A pesar de que en relación con la posición estadounidense en Medio Oriente, Romney aboga por un apoyo incondicional a Israel; las diferencias entre demócratas y republicanos en política exterior son menos extremas

15 Información de la cadena de noticias CNN sobre los temas de campaña y las posiciones de los candidatos, disponible en http:/ /edition.cnn.com/election/2012/campaign-issues.html [Consulta: 10-2-2013].

16 Extractos del discurso de Romney el 24 de julio de 2012 ante la Convención Nacional de Veteranos de Guerra Extranjera disponible en FOXNews.com http:/ foxnewsinsider.com/201/07/24/transcript-mitt-romneysremarks-at-vfw-national-convention [Consulta: 10-2-2013]. 
que las existentes en política doméstica, por ejemplo, acordando con el calendario favorecido por la OTAN para el retiro de tropas de Afganistán hacia fines de $2014 .{ }^{17}$

Resulta interesante resaltar que también las encuestas de opinión y su interpretación fueron grandes protagonistas de la campaña. En particular, la rencilla entre, los opinólogos de los medios y los periodistas contra Nate Silver y su modelo estadístico para predecir el resultado de la elección. Focalizando su atención en encuestas a nivel nacional, los medios tradicionales de comunicación favorecían una interpretación de la contienda como too close to call (demasiado reñida como para predecir quién va a ganar), ya que los sondeos marcaban una diferencia porcentual promedio de 0,2 a favor de Obama en el voto popular. ${ }^{18}$ Así, los periodistas y los pundits resaltaban la incertidumbre acerca del resultado electoral (Elving y Liasson, 2012; Jackson, 2012) contra los análisis de Nate Silver y otros expertos como Sam Wang del Princeton Election Consortium quienes, a partir de modelos que tomaban como insumos a las encuestas a nivel estatal, predecían una clara victoria de Obama (McQuaid, 2012).

Que finalmente Nate Silver y Sam Wang hayan estado en lo correcto respecto del resultado final no debería ser sorprendente ${ }^{19}$ El sistema electoral indirecto utilizado para elegir presidente en los Estados Unidos tiene como característica principal la agregación de los ciudadanos en cada estado. Esto es, en todos los estados con la excepción de Nebraska y Maine donde el reparto se hace por circunscripción electoral, la fórmula más votada se lleva todos los electores en juego. ${ }^{20}$ Además, la gran mayoría de los estados no varían su preferencia partidaria de elección en elección y suelen ser considerados distritos seguros. La contienda se define entonces en un puñado de estados conocidos como battleground o swing, que son los que se mantienen abiertos para que los gane cualquiera de los partidos. ${ }^{21}$ Por ende, son las encuestas en estos estados las que proveen la información clave para predecir el resultado final de la elección. Esto es aún más relevante si tenemos en cuenta que en cuatro ocasiones históricas el voto popular se inclinó por un ganador, pero en el Colegio Electoral se impuso el otro partido que finalmente resultó elegido. ${ }^{22}$

En suma, la campaña electoral mostró la polarización entre ambos partidos en lo concerniente a cuestiones de política interna. Tanto en los aspectos económicos como en los socioculturales, demócratas y republicanos patrocinan causas enfrentadas (por ejemplo, el derecho a la vida vs. el derecho a las mujeres a decidir sobre su propio

17 Información disponible en http://edition.cnn.com/election/2012/campaign-issues.html [Consulta: 10-2-2013].

18 Según el website Real Clear Politics, disponible en http://www.realclearpolitics.com/elections [Consulta: 10-2-2013].

19 Sam Wang predijo la elección con exactitud, mientras que Nate Silver estimó que era más probable que Florida fuera para Romney cuando en realidad el estado lo terminó ganando Obama.

20 En esto dos estados se asignan dos electores a quien haya salido primero en el estado y el resto se divide según el ganador en cada distrito electoral para la cámara baja federal.

21 En la elección de 2012, los estados que estaban indecisos era Iowa, Colorado, Carolina del Norte, Nevada, New Hampshire, Virginia, Ohio y Florida. Los dos últimos son los más relevantes debido al número de electores que eligen. Información disponible en Politico http:/ / www.politico.com/2012-election/swing-state [Consulta: 17-2-2013].

22 El candidato más votado a nivel nacional no resultó elegido 1824 (Andrew Jackson), 1876 (Samuel J. Tilden), 1888 (Grover Cleveland) y 2000 (Al Gore) (Edwards 2004). 
cuerpo en temas de salud reproductiva) o favorecen soluciones opuestas (por ejemplo, una estructura impositiva regresiva vs. una estructura progresiva). Dicha polarización pierde intensidad en relación con los asuntos externos. Puesto que, si bien con matices, ambos partidos encuentran una mayor afinidad con respecto al rol de los Estados Unidos en el mundo. El sistema electoral, sin embargo, hace que la polarización solo sea electoralmente relevante en aquellos estados que no son seguros, donde cualquiera de los dos partidos puede resultar ganador.

\section{ELECCIONES GENERALES 2012: LOS RESULTADOS ELECTORALES}

\section{Elección presidencial}

Desafiando tanto las predicciones de la literatura sobre voto económico como la de los pundits focalizados en la economía, el Partido Demócrata mantuvo la Casa Blanca ganando tanto el voto popular como el Colegio Electoral. Las elecciones del 6 de noviembre de 2012 confirmaron la reelección de Barack Obama a la presidencia de los Estados Unidos. Sin embargo, teniendo en cuenta la participación electoral, esta contienda concitó menos entusiasmo que la de 2008 en la cual Obama se impuso a John McCain. En 2012 el promedio nacional de la participación en la elección presidencial fue del 58,9 por ciento de la población elegible para votar (McDonald, 2012), mientras que en 2008 el 61,8 por ciento se había acercado a las urnas (Sullivan, 2012).

La victoria en el Colegio Electoral del candidato demócrata se tradujo en la obtención de una delegación del 62 por ciento de los electores ( 332 por sobre un total de 538). ${ }^{23}$ A pesar de que los sondeos nacionales estimaban que el voto popular iba a estar muy peleado, Obama terminó imponiéndose con el 50,9 por ciento de los votos, casi cuatro puntos por sobre la candidatura del Partido Republicano (ver Tabla 1). No solo candidaturas de los partidos Demócrata y Republicano participaron en la elección. Los partidos Libertario, Verde, de la Constitución y de la Justicia también presentaron candidatos, aunque no en todos los estados. De todas formas, el bipartidismo estadounidense es sólido y son pocos los estados en los que el número efectivo de partidos es superior a dos.

La coalición que llevó a Obama a la victoria estuvo compuesta por votantes pertenecientes a minorías raciales y étnicas: un 93 por ciento de los afroamericanos, un 71 por ciento de los latinos y un 73 por ciento de asiáticos. Además, consiguió el apoyo de 60 por ciento de los jóvenes y el 53 por ciento de las mujeres (Todd et al., 2012). ${ }^{24}$ Como se mencionó anteriormente, esto dista del perfil del votante estadounidense promedio: hombres blancos de mediana edad. Sin embargo, el perfil del votante americano promedio está cambiando en un aspecto principal: el electorado caucásico retrocede cada vez más. 
Tabla 1: Voto por partido y estado, elecciones presidenciales 2012 (totales y porcentajes)

\begin{tabular}{|c|c|c|c|c|c|}
\hline \multirow{2}{*}{$\begin{array}{l}\text { Estado } \\
\text { Alabama }\end{array}$} & \multirow{2}{*}{$\begin{array}{c}\begin{array}{c}\text { Número } \\
\text { de electores }\end{array} \\
9\end{array}$} & \multicolumn{2}{|c|}{ P. Demócrata } & \multicolumn{2}{|c|}{ P. Republicano } \\
\hline & & 795.696 & 38,4 & 1.255 .925 & 60,6 \\
\hline Alaska & 3 & 122.640 & 41,2 & 164.676 & 55,3 \\
\hline Arizona & 11 & 1.025 .232 & 44,6 & 1.233 .654 & 53,7 \\
\hline Arkansas & 6 & 394.409 & 36,9 & 647.744 & 60,6 \\
\hline California & 55 & 7.854 .285 & 60,2 & 4.839 .958 & 37,1 \\
\hline Colorado & 9 & 1.322 .998 & 51,5 & 1.185 .050 & 46,1 \\
\hline Connecticut & 7 & 905.083 & 58,1 & 634.892 & 40,7 \\
\hline Delaware & 3 & 242.584 & 58,6 & 165.484 & 40,0 \\
\hline Washington DC & 3 & 267.070 & 90,9 & 21.381 & 7,3 \\
\hline Florida & 29 & 4.237 .756 & 50,0 & 4.163 .447 & 49,1 \\
\hline Georgia & 16 & 1.773 .827 & 45,5 & 2.078 .688 & 53,3 \\
\hline Hawai & 4 & 306.658 & 70,6 & 121.015 & 27,8 \\
\hline Idaho & 4 & 212.787 & 32,6 & 420.911 & 64,5 \\
\hline Illinois & 20 & 3.019 .512 & 57,6 & 2.135 .216 & 40,7 \\
\hline Indiana & 11 & 1.152 .887 & 43,9 & 1.420 .543 & 54,1 \\
\hline Iowa & 6 & 822.544 & 52,2 & 730.617 & 46,4 \\
\hline Kansas & 6 & 440.726 & 38,0 & 692.634 & 59,8 \\
\hline Kentucky & 8 & 679.370 & 37,8 & 1.087 .190 & 60,5 \\
\hline Louisiana & 8 & 809.141 & 40,6 & 1.152 .262 & 57,8 \\
\hline Maine & 4 & 401.306 & 56,4 & 292.276 & 41,1 \\
\hline Maryland & 10 & 1.677 .844 & 62,2 & 971.869 & 36,0 \\
\hline Massachusetts & 11 & 1.921 .290 & 60,8 & 1.188 .314 & 37,6 \\
\hline Michigan & 16 & 2.564 .569 & 54,2 & 2.115 .256 & 44,7 \\
\hline Minnesota & 10 & 1.546 .167 & 52,8 & 1.320 .225 & 45,1 \\
\hline Mississippi & 6 & 562.949 & 43,8 & 710.746 & 55,3 \\
\hline Missouri & 10 & 1.223 .796 & 44,4 & 1.482 .440 & 53,8 \\
\hline Montana & 3 & 201.839 & 41,7 & 267.928 & 55,4 \\
\hline Nebraska & 5 & 302.081 & 38,2 & 475.064 & 60,1 \\
\hline Nevada & 6 & 531.373 & 52,7 & 463.567 & 45,9 \\
\hline New Hampshire & 4 & 369.561 & 52,2 & 329.918 & 46,6 \\
\hline New Jersey & 14 & 2.122 .786 & 58,3 & 1.478 .088 & 40,6 \\
\hline New Mexico & 5 & 415.335 & 53,0 & 335.788 & 42,8 \\
\hline New York & 29 & 4.018 .385 & 60,4 & 2.145 .628 & 32,3 \\
\hline North Carolina & 15 & 2.178 .391 & 48,5 & 2.270 .395 & 50,5 \\
\hline North Dakota & 3 & 124.966 & 38,7 & 188.320 & 58,3 \\
\hline Ohio & 18 & 2.827 .621 & 50,7 & 2.661 .407 & 47,7 \\
\hline
\end{tabular}




\begin{tabular}{lrrrrr}
\hline \multicolumn{1}{c}{ Estado } & $\begin{array}{c}\text { Número } \\
\text { de electores }\end{array}$ & \multicolumn{2}{c}{ P. Demócrata } & \multicolumn{2}{c}{ P. Republicano } \\
\hline Oklahoma & 7 & 443.547 & 33,2 & 891.325 & $\mathbf{6 6 , 8}$ \\
Oregon & 7 & 970.488 & $\mathbf{5 4 , 6}$ & 754.175 & 42,5 \\
Pennsylvania & 20 & 2.990 .274 & $\mathbf{5 2 , 1}$ & 2.680 .434 & 46,7 \\
Rhode Island & 4 & 279.677 & $\mathbf{6 2 , 7}$ & 157.204 & 35,2 \\
South Carolina & 9 & 865.941 & 44,1 & 1.071 .645 & $\mathbf{5 4 , 6}$ \\
South Dakota & 3 & 145.039 & 39,9 & 210.610 & $\mathbf{5 7 , 9}$ \\
Tennessee & 11 & 960.709 & 39,1 & 1.462 .330 & $\mathbf{5 9 , 5}$ \\
Texas & 38 & 3.308 .124 & 41,4 & 4.569 .843 & $\mathbf{5 7 , 2}$ \\
Utah & 6 & 251.813 & 24,8 & 740.600 & $\mathbf{7 2 , 8}$ \\
Vermont & 3 & 199.239 & $\mathbf{6 6 , 6}$ & 92.698 & 31,0 \\
Virginia & 13 & 1.971 .820 & $\mathbf{5 1 , 3}$ & 1.822 .522 & 47,4 \\
Washington & 12 & 1.755 .396 & $\mathbf{5 6 , 2}$ & 1.290 .670 & 41,3 \\
West Virginia & 5 & 238.230 & 35,5 & 417.584 & $\mathbf{6 2 , 3}$ \\
Wisconsin & 10 & 1.620 .985 & $\mathbf{5 2 , 8}$ & 1.407 .966 & 45,9 \\
Wyoming & 3 & 69.286 & 27,8 & 170.962 & $\mathbf{6 8 , 6}$ \\
Total nacional & 538 & 65.446 .032 & $\mathbf{5 0 , 9}$ & 60.589 .084 & 47,1 \\
\hline
\end{tabular}

*En negrita el ganador.

**Los porcentajes no dan 100 porque se excluyó la categoría "Otros".

Fuente: Elaboración propia a partir de datos de U.S. National Archives and Records Administration, disponibles en http://www.archives.gov/federal-register/electoral-college/2012/popular-vote. html_[Consulta: 19-2-2013].

Esto representa un problema para los republicanos que ganaron el 89 por ciento de esa franja demográfica. El otro desafío para los republicanos es su aparente incapacidad de retener el voto latino. Mientras que en 2004 George W. Bush obtuvo el 40 por ciento de apoyo latino, John McCain bajó al 31 por ciento en 2008 y Mitt Romney al 27 por ciento en 2012 (Martin, 2012). Este no es un dato menor teniendo en cuenta el creciente impacto de esta demografía en estados clave como Florida, Colorado y Nevada.

A pesar de la crisis económica, Obama logró ganar un estado golpeado por el desempleo como Florida, "en parte porque hay 190.000 hispanos y 50.000 afroamericanos más en el estado de los que había en 2008" (Martin, 2012: 1). La explicación más sencilla de la creciente alineación de los votantes hispanos en contra de los republicanos yace en las posturas firmes en contra de la amnistía a los inmigrantes indocumentados. ${ }^{25} \mathrm{Sin}$ embargo, McCain tenía una agenda más indulgente en este aspecto y tampoco logró

25 Mitt Romney llegó a proponer que el gobierno debería hacerles imposible a los inmigrantes ilegales la obtención de empleo o documentos indispensables como la licencia de conducir para incentivar que ellos mismos se "autodeportaran" (Weiner, 2012). 
Figura 1: Partido ganador en el Colegio Electoral, por estado

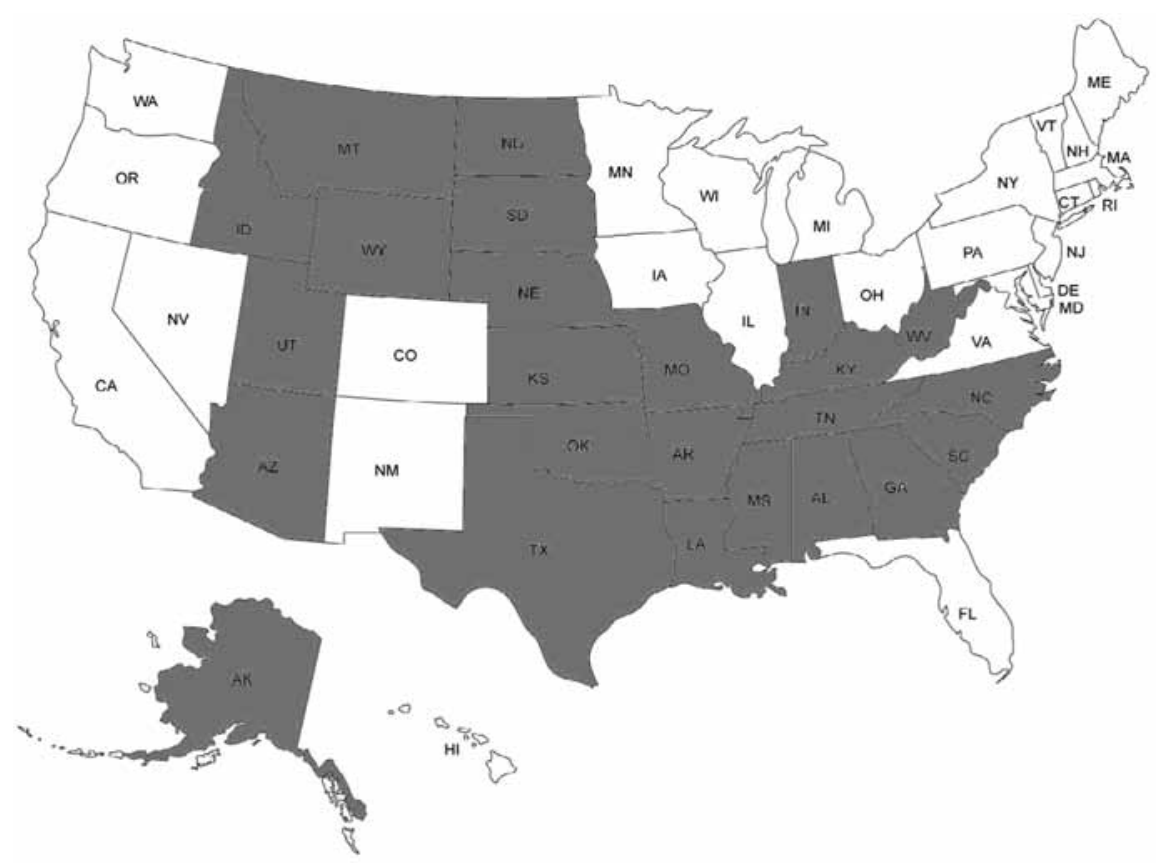

*Los estados en blanco fueron ganados por los demócratas y los sombreados por los republicanos. Fuente: Elaboración propia.

atraer al voto latino en 2008. Los sondeos de opinión indican que este voto es bastante liberal (de posiciones cercanas a una centro-izquierda moderada): "Solo el 12 por ciento de los latinos favorece una solución de recortes presupuestarios para reducir el déficit, y solo el 25 por ciento quiere derogar el Obamacare" (Yglesias, 2012). Y quizás ahí resida el principal problema de los republicanos, quienes impulsados por el Tea Party a posturas más extremas terminan perjudicándose por su propia polarización.

\section{Elecciones legislativas}

Las elecciones presidenciales no fueron las únicas llevadas a cabo en el territorio nacional, también se eligió la totalidad de la Cámara de Representantes y la mitad del Senado. Ambas contiendas se definen con un sistema electoral uninominal a simple pluralidad de sufragios. Como se discutió anteriormente, las elecciones legislativas de 2010 habían redundado en una victoria republicana que arrebató el control de la cámara baja a los demócratas. En esta ocasión, el Partido Demócrata no logró recuperar la mayoría en dicha cámara, pero recortó el tamaño de la delegación republicana que, si bien mantenía la mayoría, perdía doce escaños (Tabla 2). 
Tabla 2: Votos y escaños por partido, elecciones a la Cámara de Representantes 2012 (porcentajes)

\begin{tabular}{lrrcc}
\hline & \multicolumn{2}{c}{ VOTOS } & \multicolumn{2}{c}{ ESCAÑOS } \\
\hline P. Demócrata & 59.646 .195 & $49,00 \%$ & 201 & $46,20 \%$ \\
P. Republicano & 58.283 .047 & $47,71 \%$ & 234 & $53,80 \%$ \\
Otros & 3.414 .660 & $3,29 \%$ & & \\
\hline
\end{tabular}

Fuente: Elaboración propia a partir de datos de David Wasserman, "2012 National House Popular Vote Tracker", The Cook Political Report y de "United States House of Representatives Elections 2012", Wikipedia $^{26}$ [Consulta: 20-2-2013].

A partir de los datos expuestos en la Tabla 2 resulta evidente la discrepancia entre los votos populares y las bancas asignadas. El partido más votado a nivel nacional no logró capitalizar este caudal en escaños parlamentarios. Que ocurra esto no es algo impensado en los sistemas electorales uninominales a una vuelta (conocidos en inglés como first-pastthe-post, el primero que cruza la meta), ya que pueden producir votos desaprovechados. Dichos votos desaprovechados no son simplemente los recibidos por terceros candidatos sin chance de resultar elegidos, lo que puede ser interpretado como un resultado natural en un sistema que incentiva el voto estratégico entre los dos candidatos con más chances de ganar la elección. Como solo un voto más es suficiente para definir la elección en un determinado distrito, todos los votos que pasen este umbral son superfluos.

En el caso particular de esta elección, los analistas señalan que los esfuerzos de los republicanos por el trazado interesado de los distritos terminaron dando sus frutos en 2012 (Matthews, 2012; Wang, 2012; Millhiser, 2013). A modo de ejemplo, en dos estados clave en los que Obama resultó victorioso: "los candidatos a congresistas demócratas ganaron casi la mitad de los votos en Virginia, pero solo el 27 por ciento de las bancas, y el 48 por ciento de los votos pero solo un cuarto de los escaños en Ohio" (Palmer y Cooper, 2012). Sabiendo aprovechar las mayorías obtenidas a nivel estatal en 2010, los republicanos se lanzaron a una revolución de redistritado en estados que poseen el 40 por ciento de los distritos de la cámara baja, mientras que los demócratas controlaban las legislaturas de estados con apenas el 10 por ciento de dichos distritos (Palmer y Cooper, 2012).

Finalmente, otra razón detrás de que los demócratas hayan tenido más votos desaprovechados que los republicanos es lo que Chen y Rodden denominan unintentional gerrymandering (2012). ${ }^{27}$ Esto implica simplemente que como los demócratas tienden a concentrarse en

26 Disponibles en línea en https://docs.google.com/spreadsheet/ccc?key=0AjYj9mXEIO_QdHZCbzJocGtx YkR6OTdZbzZwRUFvS3c\#gid=0 y http:/ / en.wikipedia.org/wiki/United_States_House_of_Representatives _elections,_2012, respectivamente [Consulta: 20-2-2013].

27 El concepto de "gerrymandering no intencional" parece incluir una contradicción en sus términos, ya que gerrymandering es la forma en que se conoce al trazado interesado de los distritos electorales, pero los autores lo utilizan para connotar aquellas situaciones que producen un sesgo electoral no buscado provocadas por cuestiones exógenas relacionadas con la geografía humana (Chen y Rodden 2012; Palmer y Cooper 2012). 
zonas metropolitanas, sus votos terminan valiendo menos y siendo más superfluos que lo de los habitantes de áreas más despobladas, debido a la sobrerrepresentación electoral de estas últimas (malapportionment). Así, los autores encuentran que es la ineficiente concentración de demócratas lo que genera un sesgo a favor de los republicanos. Sin embargo, es indudable que el gerrymandering intencional también posee un impacto certero. En especial teniendo en cuenta que,

...donde los legisladores republicanos controlaron el proceso de redistritado [173 de los 435 distritos] ganaron once bancas más de las que hubieran obtenido si se mantenían las antiguas fronteras de los distritos [...] en los estados donde los republicanos controlaron el redistritado, sus candidatos ganaron el 53 por ciento de los votos y el 72 por ciento de los escaños. Los legisladores y gobernadores demócratas modificaron el trazado de 44 distritos [...] Donde controlaron el proceso, el partido ganó tres bancas más de las que hubieran obtenido y los republicanos perdieron cinco escaños. En los seis estados en que los demócratas manejaron el redistritado, sus candidatos ganaron el 56 por ciento de los votos y el 71 por ciento de las bancas (Iyer, 2012)

En lo referente a los resultados de las elecciones legislativas para la Cámara alta, los demócratas continúan manteniendo el control del Senado y aumentaron su ventaja de 51 sobre 100 a 53 senadores propios y un independiente aliado, pero no alcanzaron la mayoría necesaria para frenar los potenciales intentos de Filibuster republicanos (Tabla 3). Hasta el corriente año, en el Senado de los Estados Unidos no era posible interrumpir a un senador que tenga la palabra en la sesión, salvo que los $3 / 5$ de los miembros totales de la Cámara apoyen una moción para cerrar el debate (cloture). En casos donde ningún partido contaba con sesenta senadores, el filibuster solía ser el método empleado por la minoría como una táctica para dilatar la votación. ${ }^{28}$

De manera previsible, la elección de senadores termina siendo un asunto de alta personalización de la política. La combinación de un puesto muy visible con un sistema uninominal a una vuelta favorece la dilución de la etiqueta partidaria a favor de las características personales de cada senador. Así, resulta poco sorprendente que la mayoría de los candidatos que estaban buscando su reelección la hayan conseguido. En efecto, los quince demócratas que se presentaron a un mandato adicional fueron reelegidos, así como cinco de los siete republicanos que se postularon para un siguiente período. De todas maneras, en los casos en los que no había un senador actual como candidato, los demócratas retuvieron cinco de seis distritos y los republicanos dos de tres. ${ }^{29}$ Además de la personalización de la contienda, estos comicios senatoriales fueron históricos en lo relativo al número de mujeres elegidas: diez de las treinta y cuatro elecciones fueron ganadas por candidatas. Así, un 20 por ciento de la cámara está compuesto

28 En enero de 2013, el Senado cambió sus reglas internas, requiriéndose ahora catorce senadores (siete por la mayoría y siete por la minoría incluyendo a los presidentes de los bloques mayoritario y minoritario) para pedir una moción de cierre del debate (Krohn 2013). Sin embargo, aún es muy pronto para conocer el efecto real de esta reforma.

29 Datos de "United States Senate elections, 2012", Wikipedia, disponible en http://en.wikipedia.org/wiki/ United_States_Senate_elections,_2012 [Consulta: 23-2-2013]. 
Tabla 3: Votos por partido y estado, elecciones al Senado 2012

\begin{tabular}{|c|c|c|c|c|c|c|}
\hline \multirow{2}{*}{ Estado } & \multicolumn{2}{|c|}{ P. Demócrata } & \multicolumn{2}{|c|}{ P. Republicano } & \multicolumn{2}{|c|}{ Otros } \\
\hline & Votos & Porcentaje & Votos & Porcentaje & Votos & Porcentaje \\
\hline Arizona & 943.660 & $46 \%$ & 1.024 .459 & $50 \%$ & & \\
\hline California & 6.538 .196 & $62 \%$ & 4.081 .156 & $38 \%$ & & \\
\hline Connecticut & 815.077 & $55 \%$ & 637.857 & $43 \%$ & & \\
\hline Delaware & 265.374 & $66 \%$ & 115.694 & $29 \%$ & & \\
\hline Florida & 4.521 .534 & $55 \%$ & 3.457 .254 & $42 \%$ & & \\
\hline Hawaii & 266.435 & $63 \%$ & 159.002 & $37 \%$ & & \\
\hline Indiana & 1.268 .407 & $50 \%$ & 1.126 .832 & $44 \%$ & 146.453 & $6 \%$ \\
\hline Maine & 91.694 & $13 \%$ & 213.644 & $31 \%$ & 368.130 & $53 \%$ \\
\hline Maryland & 1.335 .706 & $55 \%$ & 642.443 & $27 \%$ & 409.785 & $17 \%$ \\
\hline Massachusetts & 1.678 .997 & $54 \%$ & 1.450 .044 & $46 \%$ & & \\
\hline Michigan & 2.732 .886 & $59 \%$ & 1.765 .548 & $38 \%$ & & \\
\hline Minnesota & 1.856 .196 & $65 \%$ & 868.977 & $31 \%$ & & \\
\hline Mississippi & 472.930 & $40 \%$ & 673.223 & $57 \%$ & & \\
\hline Missouri & 1.484 .683 & $55 \%$ & 1.063 .698 & $39 \%$ & 164.991 & $6 \%$ \\
\hline Montana & 234.465 & $49 \%$ & 215.701 & $45 \%$ & & \\
\hline Nebraska & 318.930 & $42 \%$ & 444.319 & $58 \%$ & & \\
\hline Nevada & 444.337 & $45 \%$ & 456.471 & $46 \%$ & & \\
\hline New Jersey & 1.828 .417 & $58 \%$ & 1.244 .734 & $40 \%$ & & \\
\hline New Mexico & 389.274 & $51 \%$ & 346.938 & $45 \%$ & & \\
\hline New York & 4.212 .518 & $72 \%$ & 1.561 .893 & $27 \%$ & & \\
\hline North Dakota & 160.752 & $50 \%$ & 157.758 & $50 \%$ & & \\
\hline Ohio & 2.645.901 & $50 \%$ & 2.371 .230 & $45 \%$ & & \\
\hline Pennsylvania & 2.943.289 & $54 \%$ & 2.453 .730 & $45 \%$ & & \\
\hline Rhode Island & 265.687 & $65 \%$ & 144.620 & $35 \%$ & & \\
\hline Tennessee & 700.753 & $30 \%$ & 1.496 .668 & $65 \%$ & & \\
\hline Texas & 3.183 .314 & $40 \%$ & 4.456 .599 & $57 \%$ & & \\
\hline Utah & 275.880 & $30 \%$ & 595.972 & $65 \%$ & & \\
\hline Vermont & & & 72.629 & $25 \%$ & 208.253 & $71 \%$ \\
\hline Virginia & 1.944 .992 & $53 \%$ & 1.758 .857 & $47 \%$ & & \\
\hline Washington & 1.714 .271 & $60 \%$ & 1.135 .453 & $40 \%$ & & \\
\hline West Virginia & 394.532 & $61 \%$ & 237.825 & $36 \%$ & & \\
\hline Wisconsin & 1.544 .274 & $51 \%$ & 1.377.253 & $46 \%$ & & \\
\hline Wyoming & 52.596 & $22 \%$ & 184.531 & $76 \%$ & & \\
\hline
\end{tabular}

*En negrita el ganador.

Fuente: Elaboración propia a partir de datos de CBS News, disponibles en http:/ /www.cbsnews. com/election-results-2012/senate.shtml [Consulta: 20-2-2013]. 
por senadoras, dieciséis demócratas y cuatro republicanas. Este número es superior al promedio mundial del 18 por ciento, pero aún está por debajo del promedio regional en el continente americano donde el 25 por ciento de los senadores son mujeres. ${ }^{30}$

\section{Elecciones subnacionales}

Estados Unidos es un país federal, compuesto por 50 estados en los cuales se eligen gobernador y representantes a la legislatura y al senado estatal. ${ }^{31}$ Asimismo, se eligen un gran número de oficiales subnacionales cuya naturaleza varía por estado. ${ }^{32}$ Las elecciones de gobernador en 2012 tuvieron lugar en once estados: Montana, New Hampshire, Carolina del Norte, Washington, Indiana, Delaware, Missouri, Vermont, Virginia Occidental, Dakota del Norte y Utah. En Wisconsin tuvo lugar una elección especial debido a una revocatoria de mandato que fue superada por el gobernador en ejercicio.

Tabla 4: Votos por partido, elecciones a gobernador 2012 (porcentajes)

\begin{tabular}{lcc}
\hline & P. Demócrata & P. Republicano \\
\hline Delaware & $\mathbf{6 9 , 3}$ & $\mathbf{2 8 , 6}$ \\
Indiana & 46,5 & $\mathbf{4 9 , 6}$ \\
Missouri & $\mathbf{5 4 , 7}$ & 42,6 \\
Montana & $\mathbf{4 9}$ & 47,3 \\
New Hampshire & $\mathbf{5 4 , 6}$ & 42,5 \\
North Carolina & 43,2 & $\mathbf{5 4 , 7}$ \\
North Dakota & 34,3 & $\mathbf{6 3 , 2}$ \\
Utah & 27,7 & $\mathbf{6 8 , 4}$ \\
Vermont & $\mathbf{5 8}$ & 37,7 \\
Washington & $\mathbf{5 1 , 2}$ & 48,8 \\
West Virginia & 50,5 & 45,7 \\
\hline
\end{tabular}

*En negrita el ganador

Fuente: Elaboración propia a partir de datos de Real Clear Politics, disponibles en http://www1. realclearpolitics.com/elections/live_results/governor/

Como muestra la Tabla 4, de las once contiendas, siete fueron ganadas por demócratas y cuatro por republicanos. Sin embargo, sería erróneo pretender entender estas elecciones a partir de una lógica nacional. En todos los casos en los que el gobernador en ejercicio se

30 Datos de la Unión Interparlamentaria (IPU) disponibles en http:/ / www.ipu.org/wmn-e/world.htm [Consulta: 23-2-2013].

31 Con la excepción de Nebraska que tiene un legislativo unicameral -además de no partisano-.

32 Incluyendo la elección directa de jueces, jefes de las fuerzas de seguridad y autoridades de los distritos escolares, entre otros puestos poco comunes. Por una cuestión de espacio, este anuario solamente comentará las elecciones a gobernador. 
presentaba a la reelección (Delaware, Missouri, Vermont, Virginia Occidental, Dakota del Norte y Utah) estos triunfaron en los comicios. En los casos en los que el gobernador en ejercicio estaba inhabilitado para buscar un nuevo mandato o había decidido no volver a postularse, el estado quedó en manos del candidato del mismo partido (con la excepción de Carolina del Norte que pasó de una gobernadora demócrata a uno republicano).

Un aspecto interesante de las elecciones subnacionales en Estados Unidos está dado por el sometimiento a votación de las medidas conocidas como propositions o ballot measures. Estas son iniciativas de política pública que son sometidas a referéndum y que están en vigencia en algunos estados. En 2012 las iniciativas que concitaron mayor atención fueron las que trataban sobre la legalización del matrimonio entre personas del mismo sexo y las de legalización de la marihuana. En los estados de Maine, Maryland y Washington, donde se preguntó al electorado sobre si el matrimonio igualitario debería ser legalizado, la mayoría estuvo de acuerdo con la moción. En Minnesota, una iniciativa que buscaba prohibir el casamiento entre personas del mismo sexo fue derrotada en las urnas. Finalmente, en los estados de Colorado, Washington y Massachusetts se legalizó la marihuana (en este último con fines medicinales), mientras que en Oregon se repudió la medida. ${ }^{33}$

\section{UN AÑO EN RESUMEN: CRISIS, REELECCIÓN Y POLARIZACIÓN}

El 2012 trajo en los Estados Unidos la reelección del primer presidente afroamericano en un contexto de una leve mejora de la crisis económica que viene asolando al país desde hace cinco años. Esta mejora en las condiciones económicas no parecía ser suficiente para predecir la reelección de Obama, al menos de acuerdo con la literatura acerca del voto económico. ${ }^{34}$ Sin embargo, el Partido Demócrata se impuso tanto en el voto popular como en el Colegio Electoral por un margen considerable. Esta victoria clara fue sorpresiva para quienes seguían los sondeos de opinión nacionales, pero no para las nuevas estrellas de las elecciones estadounidenses: Nate Silver y otros expertos como él que se dedicaron a estimar modelos estadísticos a partir de las encuestas estatales y de otros insumos relevantes.

Lo que explica entonces la victoria de Obama es, por un lado, la transformación del elector americano en los estados clave y el perfil de los votantes que le dieron su apoyo: minorías raciales, jóvenes y mujeres. Por otro lado, las posturas más extremas del Partido Republicano no le hicieron un favor a Mitt Romney, alienando franjas demográficas que se mostrarían como clave, tal como el voto latino. Cabe esperar que esta alteración del votante estadounidense se consolide en las elecciones venideras y de ser así, esto presentará un dilema de hierro al Partido Republicano que pareciera haber perdido la 23-2-2013].

34 En palabras de uno de sus principales exponentes: “...analizando diversas medidas, el Presidente Obama va a enfrentar una lucha por su reelección este otoño" (Lewis-Beck y Tien 2012, 625); más aún "[el modelo estadístico] sugiere que una victoria de Obama es poco probable" (Lewis-Beck y Tien, 2012: 629). 
capacidad (y probablemente también el interés) de apelar a votantes que no sean los hombres blancos no-hispanos. ${ }^{35}$

Sin embargo, las elecciones de 2012 también dejaron como lección la fuerte influencia del trazado interesado de los distritos de los representantes a la cámara baja. Así, los demócratas no lograron capitalizar su ventaja en el voto popular gracias al redistritado impulsado desde los estados por las mayorías republicanas. Por los próximos diez años, por lo menos hasta el próximo censo, las fronteras de los distritos -y la autoselección geográfica de los votantes, según Chen y Rodden (2012)- auguran que será muy difícil para los demócratas asegurarse la mayoría de la Cámara de Representantes. Este punto es muy relevante, ya que se espera que asuntos de alta visibilidad como el manejo del déficit fiscal, cuestiones sobre regulaciones con miras a desacelerar el calentamiento global, la reforma migratoria, el control de posesión de armas de asalto y el matrimonio igualitario sean tratados en el Congreso durante la segunda administración de Obama. De ser así, el futuro de la política estadounidense prevé no solo la permanencia del gobierno dividido, con los demócratas controlando la Casa Blanca y los republicanos la cámara baja del Congreso, sino además un impasse (gridlock) de fuerzas políticas polarizadas que volverá difícil la promulgación de las agendas legislativas por venir.

\section{REFERENCIAS}

Applebome, Peter y Marc Santora. 2013. "Biden Aids Governor in Push for New Gun Laws in Connecticut." The New York Times, 21 de febrero, A18.

Blake, Eric S., Todd B Kimberlain, Robert J Berg, John P Cangialosi, y John L. Beven II. 2013. “Hurricane Sandy: October 22-29, 2012 (Tropical Cyclone Report)". Edited by United States National Oceanic and Atmospheric Administration's National Weather Service.

Cannon, Carl M. . 2012. "Middle East Forces Its Way Into Presidential Race". In Real Clear Politics, 12 de septiembre. Disponible en http://www.realclearpolitics.com/articles/2012/09/12/egypt_libya_ and_the_perils_of_foreign_policy_politics_115417.html.

Chen, Jowie y Jonathan Rodden. 2012. “Unintentional Gerrymandering: Political Geography and Electoral Bias in Legislatures. Manuscrito". http://www-personal.umich.edu/ jowei/florida.pdf.

Edwards, George C. 2004. Why the electoral college is bad for America. New Haven, Conn.: Yale University Press. Elving, Ron y Mara Liasson. 2012. “NPR Poll Finds Presidential Race Too Close To Call”. In It's All Politics: NPR.org. 29 de octubre.

Greenfield, Rebecca. 2012. "The Types of People Who Voted for Obama". The Atlantic Wire, 7 de noviembre. Disponible en: http://www.theatlanticwire.com/politics/2012/11/types-people -who-voted-obama/58794/

Healy, Jack. 2013. "Colorado Gun Control Proposals Face Fight." The New York Times, 5 de febrero, A14.

Helleiner, Eric. 2011. "Understanding the 2007-2008 Global Financial Crisis: Lessons for Scholars of International Political Economy." Annual Review of Political Science, Vol 14: 67-87.

Iyer, Sundeep. 2012. "Redistricting and Congressional Control Following the 2012 Election". Edited by New York University of Law: Brennan Center for Justice http://www.brennancenter.org/sites/ default/files/legacy/Democracy/Redistricting_Post_2012.pdf.

Jackson, David. 2012. "Presidential race too close to call." USA Today, 4 de noviembre.

35 El Centro de Investigación Pew proyecta para 2050 una disminución del porcentaje de la población blanca por sobre el total de habitantes de 20 puntos y un crecimiento de 15 puntos de la población hispana (Passel y Cohn, 2008: 1). 
Keiswetter, Allen L. 2012. "The Arab Spring: Implications for US Policy and Interests". Middle East Journal, http://www.mei.edu/content/arab-spring-implications-us-policy-and-interests.

Kirkpatrick, Evron M. 1971. "Toward a More Responsible 2-Party System - Political Science, Policy Science, or Pseudo-Science." American Political Science Review 65 (4): 965-990.

Krohn, Jonathan. 2013. "What the Senate Filibuster Deal Does -and Doesn't Do". Mother Jones, 25 de enero. Lewis-Beck, Michael S. y Mary Stegmaier. 2000. "Economic determinants of electoral outcomes." Annual Review of Political Science 3: 183-219.

Liptak, Adam. 2012. "Supreme Court Upholds Health Care Law, 5-4, in Victory for Obama." The New York Times, 28 de junio, 5-4.

Lowi, Theodore J. 1983. “Toward a More Responsible 3-Party System -the Mythology of the 2-Party System and the Prospects for Reform." PS 16 (4): 699-706.

Martin, Jonathan. 2012. "Election aftermath: GOP soul-searching: 'Too old, too white, too male'?" Politico, 7 de noviembre.

Matthews, Dylan 2012. "How redistricting could keep the House red for a decade". In Wonkblog: The Washington Post , 8 de noviembre http:/ /www.washingtonpost.com/blogs/wonkblog/wp/2012/11/08/ how-redistricting-could-keep-the-house-red-for-a-decade.

McDonald, Michael. 2012. 2012 General Elections Turnout Rate. George Madison University. Available from http://elections.gmu.edu/Turnout_2012G.html.

McQuaid, John. 2012. "Three Lessons From The Nate Silver Controversy". Forbes.com , 7 de noviembre. Disponible en http://www.forbes.com/sites/johnmcquaid/2012/11/07/ three-lessons-from-the-nate-silver-controversy/.

Millhiser, Ian. 2013. “Thanks To Gerrymandering, Democrats Would Need To Win The Popular Vote By Over 7 Percent To Take Back The House". In Think Progress, 2 de enero. http:/ /thinkprogress.org/ justice/2013/01/02/1382471/thanks-to-gerrymandering-democrats-would-need-to-win-the-popularvote-by-over-7-percent-to-take-back-the-house.

Nisbet, Matthew C. 2008. "Horse Race Journalism". In Encyclopedia of Survey Research Methods, edited by Paul J. Lavrakas. Thousand Oaks, CA: SAGE Publications.

OECD. 2012. “OECD Economic Surveys: United States 2012". OECD Publishing http:/ /www.oecd.org/ unitedstates/economicsurveyoftheunitedstates2012.htm.

Palmer, Griff y Michael Cooper. 2012. "How Maps Helped Republicans Keep an Edge in the House." The New York Times, 15 de diciembre, A10.

Parties, APSA Committe on Political. 1950. "Toward a More Responsible Two-Party System: A Report of the Committee on Political Parties." American Political Science Review 44 (3): Part 2, Supplement.

Paulson, Arthur C. 2007. Electoral realignment and the outlook for American democracy, Northeastern series on democratization and political development. Boston: Northeastern University Press.

Pomper, Gerald M. 1971. “Toward a More Responsible 2-Party System What, Again." Journal of Politics 33 (4): 916-940.

Ranney, Austin. 1951. "Toward a More Responsible Two-Party System: ACommentary." American Political Science Review 45 (2): 488-499.

Rosenthal, Andrew. 2012. “Romney on FEMA, Then and Now". In Taking Time. New York: The New York Times, 1 de noviembre http://takingnote.blogs.nytimes.com/2012/11/01/romney-on-fema-then-and-now/.

Schlesinger, Arthur. 1951. "Toward a More Responsible Two-party System. A Report of the Committee on Political Parties (supplement to American Political Science Review, September, 1950)." Annals of the American Academy of Political and Social Science 274: 222-222.

Shear, Michael D. 2012. "Ryan Brings the Tea Party to the Ticket". In The Caucus: The Politics and Government Blog of the Times, 12 de agosto. New York: The New York Times.

Strassel, Kimberley A. 2012. "Why Romney Chose Ryan." The Wall Street Journal, 14 de agosto.

Sullivan, Sean. 2012. "Presidential election turnout ticked down from 2008". The Fix, The Washington Post, 8 de novimbre. Disponible en http:/ /www.washingtonpost.com/blogs/the-fix/wp/2012/11/08/ presidential-election-turnout-ticked-down-from-2008-estimate-shows/ 
Todd, Chuck, Mark Murray, Domenico Montanaro y Brooke Brower. 2012. "First Thoughts: Obama's Demographic Edge". First Read on NBCNews.com, 7 de noviembre. Disponible en: http:/ / firstread. nbcnews.com/_news/2012/11/07/14993875-first-thoughts-obamas-demographic-edge.

Wang, Sam. 2012. "The House -new, with less democracy!". In Princeton Election Consortium, edited by Sam Wang, 9 de noviembre. Disponible en: http://election.princeton.edu/2012/11/09/ the-new-house-with-less-democracy/.

Williamson, Vanessa, Theda Skocpol y John Coggin. 2011. “The Tea Party and the Remaking of Republican Conservatism." Perspectives on Politics 9 (1): 25-43.

Wolfinger, Raymond E. y Steven J. Rosenstone. 1980. Who votes? New Haven: Yale University Press.

Yglesias, Matthew. 2012. “The GOP's Hispanic Nightmare: Republicans' minority outreach problems go way, way beyond immigration". In Slate: Slate.com, 7 noviembre. Disponible en: http:/ / www.slate. com/articles/business/moneybox/2012/11/latino_vote_2012_opposition_to_immigration_doesn_ t_explain_romney_s_crushing.

Zeleny, Jeff y Jim Rutenberg. 2012. "Romney Chooses Ryan, Pushing Fiscal Issues to the Forefront." The New York Times, 11 de agosto.

Kumar, Anita y William Douglas. 2012. “Deal or no deal? White House, Congress agree on taxes but not on entire package." The Kansas City Star, 31 de diciembre.

Lewis-Beck, Michael S. y Charles Tien. 2012. "Election Forecasting for Turbulent Times." Ps-Political Science \& Politics 45 (4): 625-629.

Office, Congressional Budget. 2012. "Economic Effects of Policies Contributing to Fiscal Tightening in 2013". Reporte.

Passel, Jeffrey S. y D’Vera Cohn. 2008. “U.S. Population Projections: 2005-2050”. In Social and Demographic Trends. Washington DC: Pew Hispanic Center.

Weiner, Rachel. 2012. Trump: Mitt Romney's 'maniacal' self-deportation policy cost him minorities. In Post Politics, 26 de noviembre. Washington DC: The Washington Post.

Julieta Suárez-Cao es Licenciada en Ciencia Política por la Universidad de Buenos Aires y Magíster y Doctora en Ciencia Política por la Universidad de Northwestern (Estados Unidos). En la actualidad es Profesora Asistente en el área de Política Comparada del Instituto de Ciencia Política de la Pontificia Universidad Católica de Chile.

E-Mail: julieta.suarez@uc.cl 
